Revue internationale de l'économie sociale

Recma

La nouvelle alternative? Enquête sur l'économie sociale et solidaire, Philippe Frémeaux. Les Petits Matins, 2011, 160 p.

\title{
Arnaud Lacan
}

Numéro 322, octobre 2011

URI : https://id.erudit.org/iderudit/1020737ar

DOI : https://doi.org/10.7202/1020737ar

Aller au sommaire du numéro

\section{Éditeur(s)}

Association Recma

\section{ISSN}

1626-1682 (imprimé)

2261-2599 (numérique)

Découvrir la revue

Citer ce compte rendu

Lacan, A. (2011). Compte rendu de [La nouvelle alternative? Enquête sur

l'économie sociale et solidaire, Philippe Frémeaux. Les Petits Matins, 2011,

160 p.] Revue internationale de l'économie sociale, (322), 120-121.

https://doi.org/10.7202/1020737ar 
le Teikei est un "contrat moral qui pourrait être qualifié de léonin à l'égard des consommateurs, sur qui semble reposer l'essentiel des obligations". Gilles Maréchal propose également une lecture transversale autour de plusieurs thématiques : la relation et la transaction; le commerce et la production ; l'aliment et le produit ; le contrôle social et le contrat ; l'échange et l'engagement. Selon l'auteur, l'individu recherche le bonheur au sein du Teikei et la citoyenneté dans l'Amap. Lobjectif pour la collectivité serait l'harmonie dans le cas du Teikei et la justice pour l'Amap.

\section{Les fruits et les graines}

Dans le dernier texte, qui ne se veut pas conclusif, mais plutôt un commentaire des éléments marquants, Marc Humbert, économisteà l'université de Bretagne-Rennes 2, complète tout d'abord la contextualisation en enrichissant le terreau dans lequel sont nées et se sont développées ces deux formules. C'est ainsi qu'il souligne "queelles sont toutes deux issues de la vitalité de la société, elles en sont les fruits". Mais il relève également des questions, en particulier sur la capacité transformatrice de toutes ces expériences, «chacune dans un concret micro-local, quasi individuel » avec le nécessaire changement d'échelle. Comment peuvent-elles "transformer la société tout entière, et tout d'abord [...] renverser la tendance jusqu' ici dominante de l'évolution de l'agriculture "? Pour Marc Humbert, il faut associer d'autres types d'organisations " plus adaptées pour aider à développer une agriculture et une économie différentes, comme celles présentées dans la premiere partie de l'ouvrage (magasins collectifs, coopératives, associations)». La question de la participation et celle de la confiance sont liées. L'auteur suggère ainsi le développement des "systèmes participatifs de garantie " (SPG), alternatives aux labels et certifications. Les SPG sont "des systèmes d'assurance qualitéancrés localement. Ilscertifient les producteurs sur la base d'une participation active des acteurs concernés et sont construits sur une base de confiance, de réseaux et d'échanges de connaissances ". Pour Marc Humbert, on est ici dans " une logique de coopération pour faire progresser le bien-être de tous collectivement et non le contrôle pour faire fonctionner la compétition de type industriel ". Et de conclure que "toutes ces nouvelles formes de vente directe témoignent d'un renouveau de vitalitésociales et quielles sont à la fois les fruits et les graines pour son futur ".

Cet ouvrage est bien sûr destiné à toutes celles et tous ceux qui souhaitent agir pour une autre agriculture, mais il constitue aussi une excellente analyse des processus d'innovation (sociale), avec de nombreux exemples français, de tentative de transformation sociétale et de constitution d'un mouvement. Dans le contexte français, il pousse à réfléchir sur l'ordre des facteurs...

\section{Rémi Laurent}

\section{La nouvelle alternative?}

\section{Enquête sur l'économie sociale et solidaire Philippe Frémeaux. Les Petits Matins, 2011, $160 p$.}

Pour répondre à une question aussi importante, il fallait bien une enquête. Une enquête sérieuse, sans concession et qui fasse apparaittre les atouts ainsi que les faiblesses del'économie sociale et solidaire. C'est dans cet esprit que Philippe Frémeaux, président et éditorialiste de la coopérative qui édite Alternatives économiques, mais aussi chroniqueur très en vue à la radio ou à la télévision, propose une exploration de l'univers parfois mal connu, souvent idéalisé, toujours compliqué de l'économie sociale et solidaire. Dans chacun de ses dix chapitres, sa plume fait mouche et son analyse lucide servie par un ton parfois sarcastique mais jamais désenchanté nous éclaire sur les grandes problématiques de ce continent économique à qui d'aucuns promettent un grand soir en forme d'alternative à l'économie capitaliste.

Après avoir rappelé les composantes del'économie sociale et solidaire (ESS) et ses origines, l'auteur rappelle (chapitre 1) que cette dernière est née de la nécessité de répondre aux besoins sociaux. C'est d'abord une économie de la réaction nécessaire et de la construction collective d'un avenir meilleur. Mais les structures de l'économie sociale et solidaire, lorsqu'elles grandissent, peuvent perdre 
de vue leur projet originel et se laisser aller à une banalisation dangereuse parce qu'elle porte les germes de la récupération, voire de l'instrumentalisation (chapitre 2). Ce risque est d'autant plus grand que l'ESS n'est pas très visible (chapitre 3) et n'a que très rarement l'occasion de marquer les esprits de nos concitoyens - au-delà des initiés et des militants -, perdant ainsi toute possibilité de revendiquer « le monopole du sens ».

Ce voyage en terre d'économie sociale et solidaire nous emmène ensuite du côté des pratiques de gouvernance (chapitre 4) et livre une analyse juste et sans concession du fameux principe de contrôle démocratique des organisations, avant de pousser plus avant l'exploration des structures associatives (chapitre 5), dont nous savons bien qu'elles représentent aujourd'hui la plus grande vivacité et la plus vigoureuse des dynamiques pour l'ESS. La gouvernance des entreprises est bien, selon Philippe Frémeaux, un point clé de leur efficacité et de leur crédibilité, que cela se joue au niveau du rôle des managers dans la garantie et la transmission des valeurs (chapitre 6) ou au niveau plus large du politique (chapitre 7) et de la gestion des parties prenantes.

Enfin, avec la lucidité et la liberté de ton qui le caractérisent, l'auteur dresse une liste sans concession des faiblesses de l'économie sociale et solidaire (chapitre 8) qui la conduisent à ne s'affirmer que très difficilement en tant que mouvement social, avant de nous proposer (chapitre 9) une piste de solution, celle du changement d'échelle vers une extension allant plus à l'encontre de la demande sociale en mettant en œuvre une activité économique plus solidaire et plus soutenable. Pour achever son propos, Philippe Frémeaux livre sa vision prospective de l'ESS (chapitre 10) en rappelant que l'objectif de celle-ci devrait être d'œuvrer à la transformation de nos modes de production et de consommation dans un sens plus démocratique et plus respectueux de notre environnement de vie sociale. L'économie sociale et solidaire, nous rappellet-il, n'a pas vocation à devenir hégémonique. Elle n'est pas un modèle qui s'impose à tous, la fameuse alternative au capitalisme, mais un exemple qui, en dépit de ses imperfections, démontre en actes la possibilité d'imposer des logiques économiques fondées sur d'autres logiques que celles du simple profit.

La nouvelle alternative est un ouvrage percutant parce que pertinent. Son auteur y fait une analyse froide et objective de l'économie sociale et solidaire. Chacun en sort plus riche. Les sceptiques y découvriront la capacité du secteur à se questionner, à se remettre en cause pour mieux identifier les chemins de son propre progrès. Les euphoriques de l'ESS y trouveront quelques vérités bonnes à dire, même si elles sont parfois politiquement incorrectes parce qu'elles piquent le militant au cœur de ses convictions. C'est justement ce qui est bon.

\section{Arnaud Lacan}

\section{Manifeste pour une économie plus humaine: construire le social-business} Muhammad Yunus. Ed. J.-C. Lattès, 2011, ISBN 978-2-7096-3550-9, $304 p$.

A l'heure où la crise bouche les horizons, à moins qu'elle ne libère l'imagination en nous débarrassant de nos chimères, nous ne saurions trop remercier M. Yunus de vouloir nous faire rêver. Je confesse cependant que, bonne ou mauvaise volonté de ma part, je ne sais, je ne suis pas parvenu à me laisser emporter. Il s'agit pourtant d'un livre "grand public", structuré en neuf parties, qui alternent utopie, description de réalisations concrètes, analyses juridiques ou économiques, bilans d'expériences.

\section{Appel messianique à en finir avec la pauvreté} Il est difficile de décrire objectivement les ouvrages de M. Yunus, car ils sont si typés qu'ils ne peuvent laisser indifférent. Son ton a un aspect messianique auquel nous ne sommes plus habitués, comme en attestel'histoire prospective d'un musée de la pauvreté où, dans quelques décennies, pour peu que le social business soit reconnu et se développe, on viendra admirer avec candeur les images d'un temps où la pauvreté 